\title{
The effect of figural manipulations on brightness differences in the Benary cross
}

\author{
Mark Vergeer, Rob van Lier \\ Radboud University Nijmegen, Donders Institute for Brain, Cognition and Behaviour, PO Box 9104, \\ 6500 HE Nijmegen, The Netherlands; e-mail: m.vergeer@donders.ru.nl \\ Received 28 July 2009, in revised form 29 March 2011
}

\begin{abstract}
The Benary cross is a classical demonstration showing that the perceived brightness of an area is not solely determined by its luminance, but also by the context in which it is embedded. Despite the fact that two identical grey triangles are flanked by an equal amount of black and white, one of the triangles is perceived as being lighter than the other. It has been argued that the junctions surrounding a test area are crucial in determining brightness. Here, we explored how different aspects influencing perceptual organisation influence perceived figurebackground relations in the Benary cross and, with that, the perceived brightness of the triangular patches in our stimuli. The results of a cancellation task confirm that the alignment of contours at junctions indeed has a strong influence on an area's brightness. At the same time, however, the Benary effect is also influenced by the overall symmetry of the cross and its orientation.
\end{abstract}

\section{Introduction}

The brightness of an area is not solely determined by its own luminance, but also by other luminances present in the same visual scene. This becomes clear in demonstrations of simultaneous brightness contrast (SBC), which show that a homogeneous grey area looks lighter when surrounded by a homogenous dark area than when surrounded by a homogeneous light area. According to the ratio rule of brightness (Wallach 1948) the difference in brightness of two test areas of the same luminance is determined by the amount of black and white surrounding each of these test areas. However, in the literature there are several examples of situations in which this rule is violated, meaning that the brightness relation of two test areas is different from what the ratio rule would predict solely on the basis of the surrounding amounts of black and white in the visual scene (eg Adelson 1993; Benary 1924; Oh and Kim 2004; White 1979). These examples all show that the figural properties of a scene influence the perceived brightness of the separate parts of this scene. One of the best-known demonstrations is the Benary cross, discovered by Max Wertheimer and studied further by Benary (1924), which is presented in figure 1.

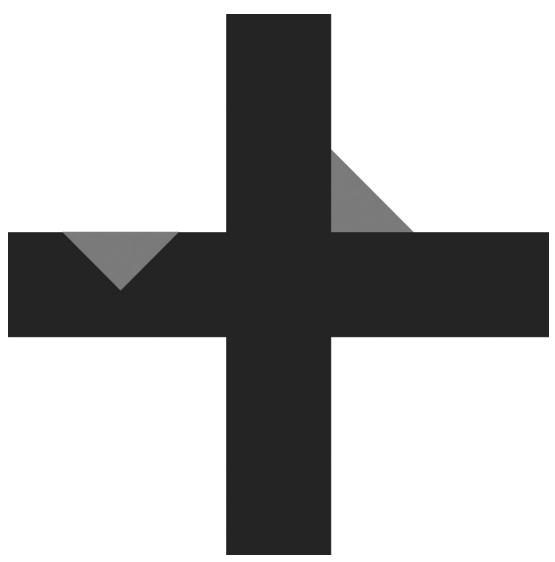

Figure 1. The Benary cross. Although both grey triangles are surrounded by the same amount of black and white, the left one appears to be lighter than the right one. 
Attempts to unravel the mechanisms underlying brightness perception can broadly be divided into theoretical accounts relying on low-level mechanisms operating on early visual input and accounts in which a more conceptual approach is taken. Some recent low-level brightness models based on multiscale spatial filtering have been shown to be quite successful in predicting brightness perception from the 2-D physical image. Blakeslee et al's ODOG model is a modification of their DOG model, with the difference that the ODOG model uses anisotropic instead of isotropic filters and pools its outputs nonlinearly. This model is, for instance, capable of predicting the direction of the Benary effect (Blakeslee and McCourt 2001) and White's effect (Blakeslee and McCourt 1999), although for the latter the model's predictive success varies for the different versions of the effect. The ODOG model has been extended by Robinson et al (2007) by constraining response normalisation to local receptive fields, which increased the effectiveness of the model for several versions of White's effect. The effectiveness of these models supports the idea that spatial filtering operations early in the visual system play an important role in brightness perception. Although these models relying on low-level mechanisms are capable of predicting the direction of several brightness effects, in some cases they seem to be less satisfactory when it comes to predicting the effect size of brightness effects as reported in the literature. Especially when it comes to the Benary effect, both the ODOG and the FLODOG model are capable of predicting only a small proportion of the effect size measured in human beings (see Robinson et al for an overview of predicted effect sizes of the different models for several brightness illusions).

Perception of brightness has been linked to the concepts of perceptual organisation and surface perception. A common argument is that the brightness of an area is to a large extent determined by the luminance of a reference surface. Gilchrist et al (1999) pointed out that the visual system has to tie relative luminance values extracted from the retinal image to specific perceived brightness values. They developed their anchoring theory which is based on the idea of both local and global anchoring of luminance values, in which the highest luminance is automatically assigned the colour white. Within a frame of reference, either globally or locally defined, a certain surface will have the same brightness, regardless of its exact position in this area. In other words, the brightness of an area is computed relative to its surroundings within a specific framework. This framework is determined by the spatial layout of an image and defined by Gilchrist et al as a group of surfaces that belong to each other, more or less. A somewhat different suggestion has been made by Anderson (1997, 2003). In his scission account on brightness perception he proposes that, within a local framework, scission can occur between a near-transparent surface and a more distant underlying surface. A bit simplified, this means that when a grey target is perceived on a light surface, the target is decomposed into a light and a dark part with the light part being attributed to the light-background surface, and the dark part to the target itself. For a grey target on a dark surface the decomposition process is similar, only now the dark part is attributed to a background layer and the light part to the target.

In both anchoring theory and scission theory there is an important role for contour junctions. According to scission theory, decomposition into multiple layers can be initiated by contrast variations that occur along the top of a T-junction. In anchoring theory, the perceptual quality of belongingness within a framework influences the anchoring of a surface within this specific framework. T-junctions are an important grouping factor influencing this perceptual quality. The influence of the specific alignment of different luminance areas on perceived brightness has been acknowledged throughout the lightness/brightness literature. Zaidi et al (1997), for instance, manipulated perceived depth relations by presenting monocular 3-D images. The important role of T-junctions was demonstrated with several examples, both in 2-D images and in monocular 3-D images. 


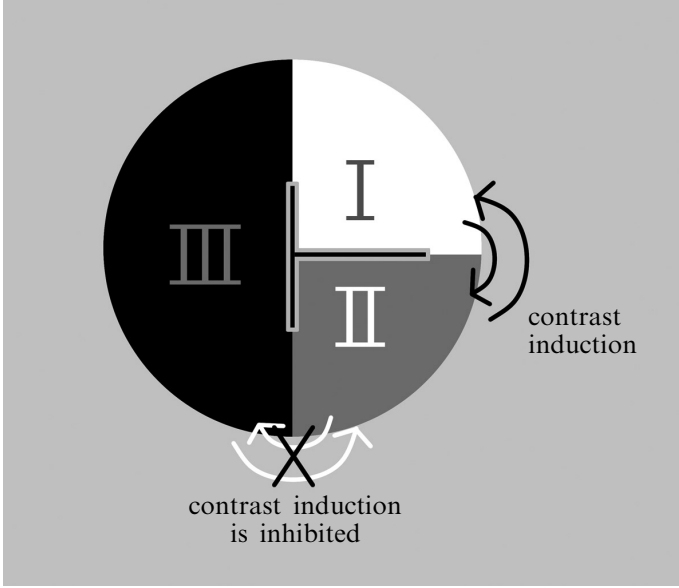

Figure 2. It is suggested that contrast induction occurs between the areas on both sides of the stem of the T-junction (I and II), while it is inhibited between the area topping the T-junction (III) and the grey area (II).

In the latter case, it was found that the position of the different luminance areas relative to the T-junction is more important in determining perceived brightness than the depth plane in which the areas are perceived. The idea is that contrast induction occurs between the areas adjacent to the stem of the T (areas I and II in figure 2), but that a similar contrast induction between the area on top of the $\mathrm{T}$ (area III) and the two other areas (areas I and II) is inhibited, as a result of the collinearity of the contours. Todorović (1997) also emphasised the importance of T-junctions in determining brightness and, following similar principles as Zaidi et al, he argued that the brightness is predominantly a function of the ratio of its luminance and the luminance of collinear regions, if an area shares its borders with several other regions whose corners involve T-junctions. Furthermore, it has been argued that T-junctions function as signalling the presence of an occlusion relationship (eg Anderson 1997). According to this argument, the area on top of the T-junction (area III in figure 2) occludes the two other luminance areas. Todorovic demonstrated that slightly shifting the test areas horizontally in White's illusion leads to drastic changes with regard to the junctions, but apparently they do not produce similarly drastic changes in brightness. In addition, Howe (2005) showed that removing the junctions in White's effect does not lead to a significant reduction of perceived brightness differences. These findings are clear indications that the presence of T-junctions in the retinal image is not in itself the cause of brightness effects, but that, as argued earlier, T-junctions are an important factor in determining perceived figure-ground relations. Possibly, grouping of a test area and a background surface could lead to contrast induction or scission between both layers, in line with the anchoring theory (Gilchrist et al 1999) and scission theory (Anderson 1997, 2003), respectively. This is in line with the idea that belongingness or grouping relations play an important role in brightness perception, as underlined by Agostini and Proffitt (1993). They facilitated belongingness by means of perceptual grouping due to common fate, figural alignment, or a combination of both. For all manipulations, they found that a grey target shape belonging to a similar group of black shapes appeared darker than a grey target shape of equal luminance belonging to a group of similar white shapes. Furthermore, Agostini and Galmonte (2002) demonstrated that the effect caused by global organisational properties of a configuration can overcome the effect of low-level lateral inhibition in determining brightness, which supports the idea that global grouping relations play a role in determining brightness. 


\subsection{The Benary cross and figure-ground segregation}

Since the Benary cross was created in an area dominated by the Gestalt tradition, it may not come as a surprise that the effect was originally presented as a demonstration that perceptual organisation influences local brightness perception. The left triangle is commonly perceived as lying on top of the cross, while the right triangle is perceived as lying on the white background, juxtaposed ${ }^{(1)}$ to the cross. In the latter case the triangle and the cross are perceived to be positioned next to each other, in the same depth plane. With the assumption that each area is contrasted with the background it is perceived on, it could be explained that the left triangle is perceived as being lighter than the right one. Differences between the two triangles in terms of local junctions have been argued to be an important factor in causing the differences in perceived figure-ground relations between the triangles. In the literature, the role that grouping and segregation processes can play in brightness perception has been demonstrated.

By means of stereoscopic presentation, Coren (1969) varied the perceived depth relations of the cross and the triangles, thus manipulating the perceived figure-ground relations. He found that the brightness of each triangle depended on the luminance of the underlying surface on which it was perceived. In one condition, the triangle superimposed on the cross was presented stereoscopically as if it were in the same depth layer as the white background, with the cross presented in a closer layer. So it looked as if this triangle was seen through a triangular hole in the cross. As a result of this manipulation, the triangle was perceived to be significantly darker than when it was presented in the same layer as the cross. For the juxtaposed cross, no such difference was perceived. These findings are in line with the findings by Zaidi et al (1997), who also showed that the brightness of a target area is influenced by the luminance of the surface on which it is perceived, independently of the perceived depth relations between the target and the background surface.

\subsection{The present study}

Literature has suggested a role for perceptual organisation in brightness perception (eg Agostini and Galmonte 2002; Agostini and Proffitt 1993; Vergeer and van Lier 2010). The aim of the present study was to explore how different aspects influencing perceptual organisation influence perceived figure-background relations in the Benary cross, and with that the perceived brightness of the triangular patches in the configurations. We have constructed several modified versions that change the perceived figure-ground organisation within the configuration. Below, we describe a selection of the configurations used in the experiment.

First we manipulated the shape of the cross (see figure $3 b$ ). The ambiguity in figureground relations is increased by making small triangular indentations to each arm of the cross. Note that, as regards contour junctions with the areas surrounding the triangles, there are no differences between the original Benary cross and our modified version of it. We do not expect the right grey triangle to be perceived differently from how it is perceived in the original cross. It is still seen as lying on the white background. The left (previously superimposed) grey triangle, however, can now more easily be perceived in two possible ways. As a result of a local analysis of the junctions, this triangle would still be perceived as lying on the cross. Taking into account that we have a preference for perceiving symmetrical visual shapes, an interpretation becomes plausible in which the left grey triangle is perceived as being juxtaposed to the cross at one of the indentations made to the cross. Combining both arguments, perceived

(1) When we use the term 'juxtaposed' to express the relative position of two surfaces, we allude to an interpretation in which one surface is positioned next to another surface in the same depth plane, without occlusion of any of their parts. This interpretation is opposed to a 'superposition' of surfaces, in which the surfaces are interpreted as being in different depth planes, where one surface is occluding part of the other surface. 


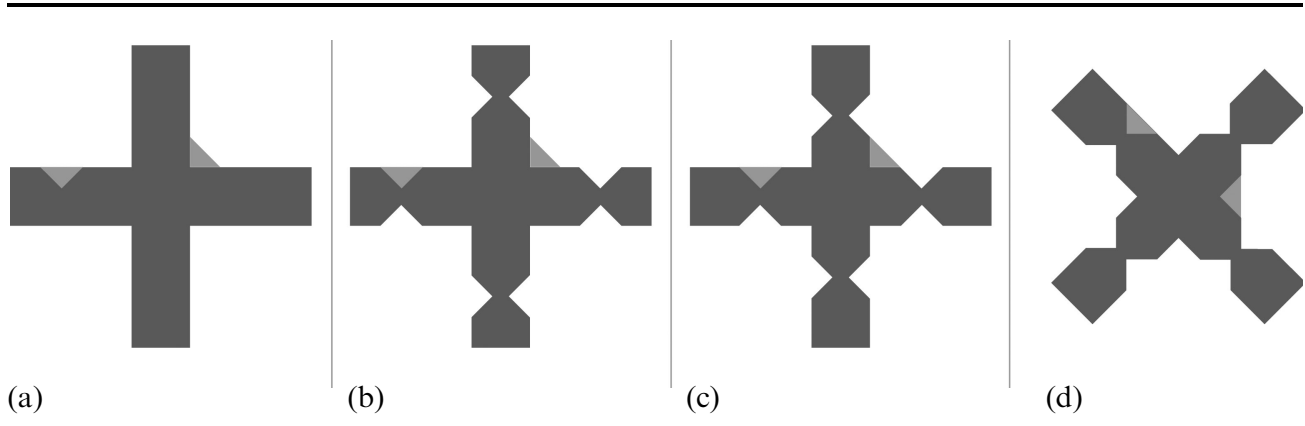

Figure 3. Examples of stimulus configurations used to study brightness differences between the left and right triangle.

figure-ground relations in this configuration are expected to be more ambiguous than in the previous manipulation. That is why we expect a reduction of brightness differences compared to the original Benary effect.

The example shown in figure $3 \mathrm{c}$ is to a large extent the same as the configuration in figure $3 b$, except for one crucial difference. The indentations made to the cross in figure $3 \mathrm{~b}$ are now shifted towards the middle of the cross until their contours are aligned with the hypotenuse of the right triangle. As a result, differences between the two triangles in contour junctions with adjacent areas are overcome. In line with the literature, which has suggested an important role for T-junctions in determining figure-ground relations, we again expect a reduction of brightness differences between the triangles. If we still find brightness differences in any direction, these differences could be the result of a preference for one specific global interpretation. The original Benary cross (figure 1) leads to a relatively unambiguous percept of a cross, consisting of a horizontal and a vertical bar crossing each other. As argued, the indentations made to the cross already increased the ambiguity of this interpretation in our first manipulation (figure $3 b$ ), but in this second manipulation this ambiguity is even further increased. The alignment of the contours of the indentations made to the cross with the hypotenuse of the right triangle increases the plausibility of an interpretation in which the dark-grey cross-like figure continues behind this right triangle. This interpretation would lead to a reversal of figure-ground relations, in which the right triangle would now be perceived as lying on top of the dark-grey central figure. With the assumption that the grey triangles are contrasted with the background on which they are perceived, this would lead to a lighter/brighter perception of the right grey triangle compared to figures $3 \mathrm{a}$ and $3 \mathrm{~b}$.

Next, we manipulated the orientation of the Benary cross and all previously described variations of it. All variations were rotated clockwise by $45^{\circ}$ (see figure $3 \mathrm{~d}$ for an example). This rotation results in different contours in the configuration that could become more salient. The contours of the original cross are now diagonal and the contours that come to existence by introducing the indentations to the cross are now either horizontal or vertical, just as the hypotenuse of the right grey triangle. Given these changes, it might very well be that, after rotation of the figure, the plausibility is increased that the left grey triangle is perceived as juxtaposed to the cross, whereas the right grey triangle is perceived as superimposed on the cross figure. In this case, we predict that the right triangle would become perceptually lighter than in the configurations before rotation.

\section{Experiment}

\subsection{Method}

2.1.1 Participants. Sixteen healthy volunteers (aged $17-33$ years, mean age 21.7 years) participated in this experiment. They were all students at the Radboud University Nijmegen, and naive with respect to the experimental questions. All participants had normal or corrected-to-normal vision and received course credit for participation. 
2.1.2 Design and stimuli. In our experiment we used a total of 12 configurations, which are all shown in figure 4 . Starting point was the original Benary cross. The cross was modified in different ways:

(i) Presence of indentations in the cross (indentations).

(ii) The position of the indentations/triangles (position).

(iii) The orientation of the figure (orientation).

Indentations has the following two levels: indentations absent and indentations present. Position has three levels: the left triangle with indentations positioned relatively on the outside of the arms of the cross; the left triangle and the indentations positioned

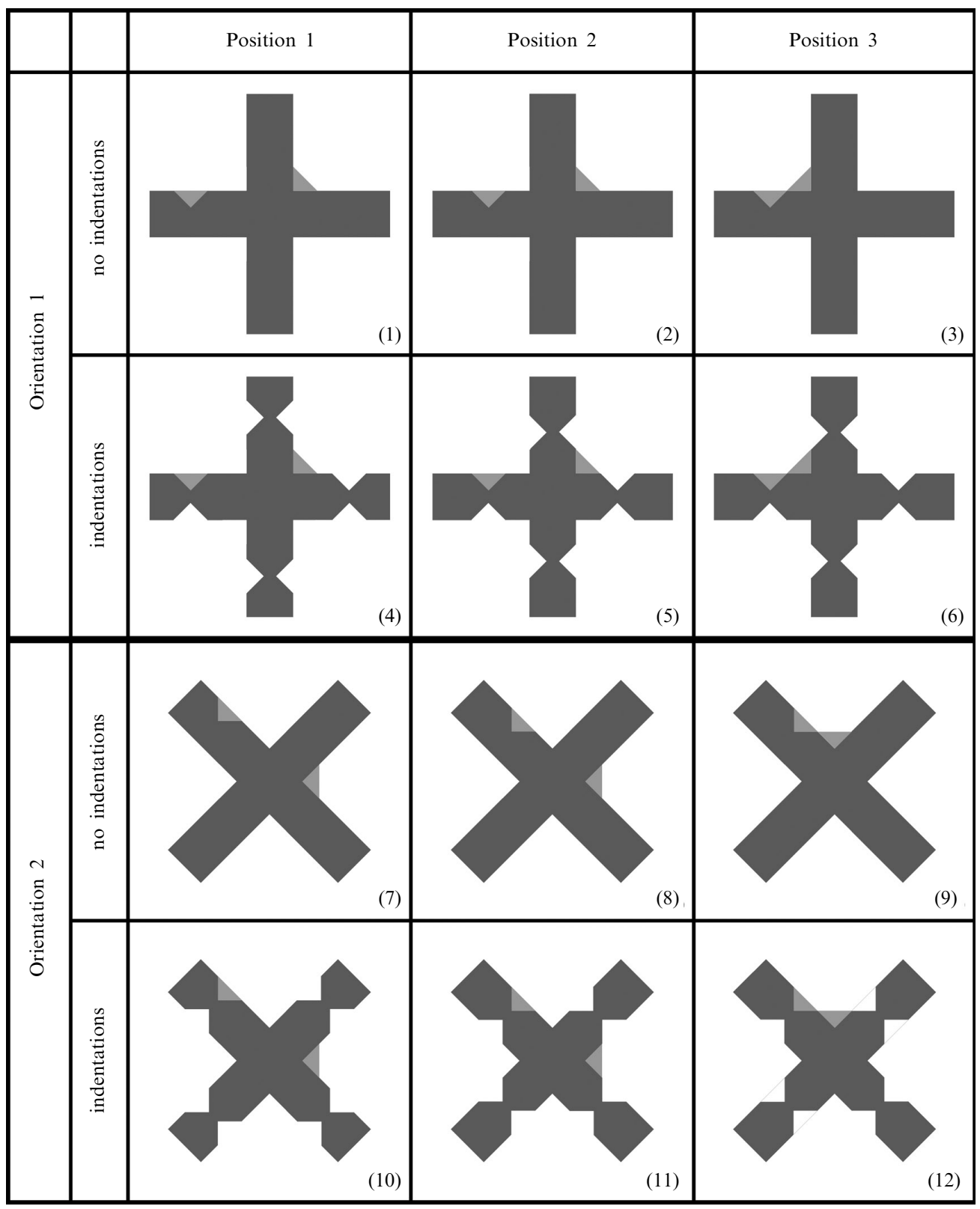

Figure 4. The stimuli used in our experiment. The crosses in the examples in this figure are somewhat darker than in the actual experiment, with the aim of increasing the visibility of the test triangles. 
more towards the centre of the cross, in such a way that the hypotenuse of the right triangle is aligned with the contour of two of the indentations; the right triangle was repositioned in such a way (mirrored across the $y$-axis) that its hypotenuse was aligned with the right cathetus of the left triangle. Orientation also has two levels: the cross positioned in an upright position (as the original Benary cross) and the cross rotated by $45^{\circ}$. These three factors combined lead to the 12 manipulations of the Benary cross displayed in figure 4, which are the stimuli used in this experiment. Note that configurations 1 and 2 are rather similar, since for these configurations the repositioning of the left triangle did not result in any significant changes. This is also the case for configurations 7 and 8. In addition, the positional change between levels 1 and 2 is rather different from the positional change between levels 2 and 3 . Therefore, with respect to the positional variations we mainly focus on the individual manipulations. In our discussion, we elaborate on the effect that the positional changes have on the local junctions and symmetry of possible interpretations of the configurations as a whole.

2.1.3 Task and procedure. In each trial, one of the configurations described above was presented on the screen at a viewing distance of $60 \mathrm{~cm}$. The cross had a luminance of $32.53 \mathrm{~cd} \mathrm{~m}^{-2}$ and consisted of two orthogonal arms $16.7 \mathrm{~cm}$ long and $3.3 \mathrm{~cm}$ wide. The grey triangles had a base (hypotenuse) of $2.5 \mathrm{~cm}$ and a height of $1.2 \mathrm{~cm}$. As a result, the crosses comprised a visual angle of $15.8 \mathrm{deg}$ and $13.2 \mathrm{deg}$, for the original orientation and the rotated versions, respectively. One of the triangles in this configuration had a grey value of 167 , which corresponded to a luminance of $43.41 \mathrm{~cd} \mathrm{~m}^{-2}$. The other triangle started with a random grey value. The left and the right triangle started at a random grey value, both in half of the trials. Stimuli were presented on a white background with a luminance of $99.9 \mathrm{~cd} \mathrm{~m}^{-2}$. The experiment was run on a PC-Pentium-III configuration, and stimuli were presented on a CRT monitor. Colorshop 2.6/monitor optimizer (X-Rite Inc.) was used for monitoring calibration and luminance measurements.

Participants were instructed to "adjust the luminance of this latter triangle, until both triangles appear to have equal brightness", with the triangles indicated as 'A' and 'B', respectively. By using four response keys on a standard keyboard the grey value could be adjusted in steps of respectively $-10,-1,+1,+10$. As soon as the participant pressed one of these four buttons, the letters ' $A$ ' and 'B' disappeared from the screen, so that they did not influence the brightness of the triangles. When the brightness of the two triangles was perceived as being equal, participant had to press the 'enter' button to continue with the next trial. Each configuration was presented in 12 trials.

\section{Results}

In each trial, participants had to match the brightness of the two triangles. We took the difference in luminance between the two triangles after brightness matching as measure for the brightness effect of each particular configuration. Subsequently, we calculated the mean luminance difference between the two grey triangles for each configuration. On these luminance differences, we first performed a repeated-measures ANOVA in which all three main effects were significant (indentations: $F_{1,15}=72.4, p<0.001$; position: $F_{2,14}=22.6, p<0.001$; orientation: $\left.F_{1,15}=33.5, p<0.001\right)$. All 2-way interactions were significant as well (indentations $\times$ position: $F_{2,14}=11.8, p<0.005$; indentations $\times$ orientation: $\quad F_{1,15}=10.3, \quad p<0.01 ; \quad$ position $\times$ orientation: $\quad F_{2,14}=14.5$, $p<0.001)$. There was no significant 3 -way interaction. The average measured luminance differences for each level of each factor are presented in figure 5.

Let us now focus on the various configurations and on the effect of each specific manipulation when transforming one configuration into the other configuration. First, we performed $t$-tests on each configuration. The effects of each separate configuration 


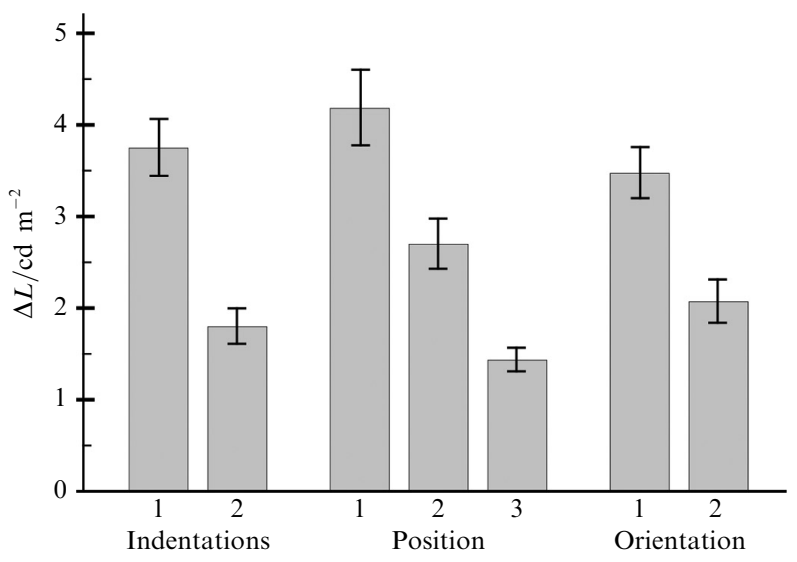

Figure 5. Main effects of the indentations, the positioning of the triangular patches and indentations (if present), and the orientation of the configuration, respectively. As can be seen, lightness differences, $\Delta L$, between the two triangles became smaller as a result of manipulating the configuration on each of the factors.

Table 1. Results on the cancellation task.

\begin{tabular}{llll}
\hline $\begin{array}{c}\text { Configuration } \\
\text { (see figure 4) }\end{array}$ & $\begin{array}{l}\text { Relative grey value } \\
\text { (left-right) }\end{array}$ & $\begin{array}{l}\Delta L / \mathrm{cd} \mathrm{m}^{-2} \\
(\text { left }- \text { right) }\end{array}$ & SEM \\
\hline 1 & 6.95 & $3.91 * *$ & 0.374 \\
2 & 6.45 & $3.62 * *$ & 0.366 \\
3 & 4.86 & $2.72 * *$ & 0.218 \\
4 & 6.03 & $3.38^{* *}$ & 0.332 \\
5 & 2.61 & $1.45^{* *}$ & 0.233 \\
6 & 2.90 & $1.60 *$ & 0.366 \\
7 & 6.30 & $3.53 * *$ & 0.410 \\
8 & 5.27 & $2.94 * *$ & 0.356 \\
9 & 2.28 & $1.26 * *$ & 0.262 \\
10 & 4.62 & $2.57 * *$ & 0.352 \\
11 & 1.14 & $0.63 *$ & 0.150 \\
12 & -1.82 & $-0.99 * *$ & 0.182
\end{tabular}

Note: Luminance differences $(\Delta L)$ after cancellation of brightness differences between the left and the right triangle by the participant. These luminance differences are taken as measure for the relative perceived brightness differences in corresponding configurations with triangles of equal luminance. $* \Delta L$ is significant with $p<0.005 ; * * \Delta L$ is significant with $p<0.001$.

as well as the statistics of the performed $t$-tests can be found in table 1 . As can be seen, for all configurations luminance differences $(\Delta L)$ were significant after matching the brightness of the two triangles. Note that the effect of configuration 12 is significant in the opposite direction compared to the effects of all other configurations.

Next, we compare the brightness effects evoked by the separate manipulations. There are several ways of step-by-step alteration of the original Benary cross (configuration 1) into its final version (configuration 12), in which the cross is manipulated on all specified factors. By going from configuration 1 to configuration 12 in such a way that just one manipulation occurs at a time, six different routes are possible. Two of these routes can be excluded (those with the transition from stimulus 1 to stimulus 2 and from stimulus 7 to stimulus 8), as they were added for reasons of factorial balance. The remaining four routes from stimulus 1 to stimulus 12 are shown in figure 6 . As can be seen, all routes show a rather similar pattern in which each further disruption of the original configuration leads to a further reduction of the original effect.

In the following, we will focus on the manipulations as shown in figure 6 . We first compared the brightness effect in the original cross with the effect after making indentations in the cross (referred to as s1), which illustrates the influence of the shape of the 

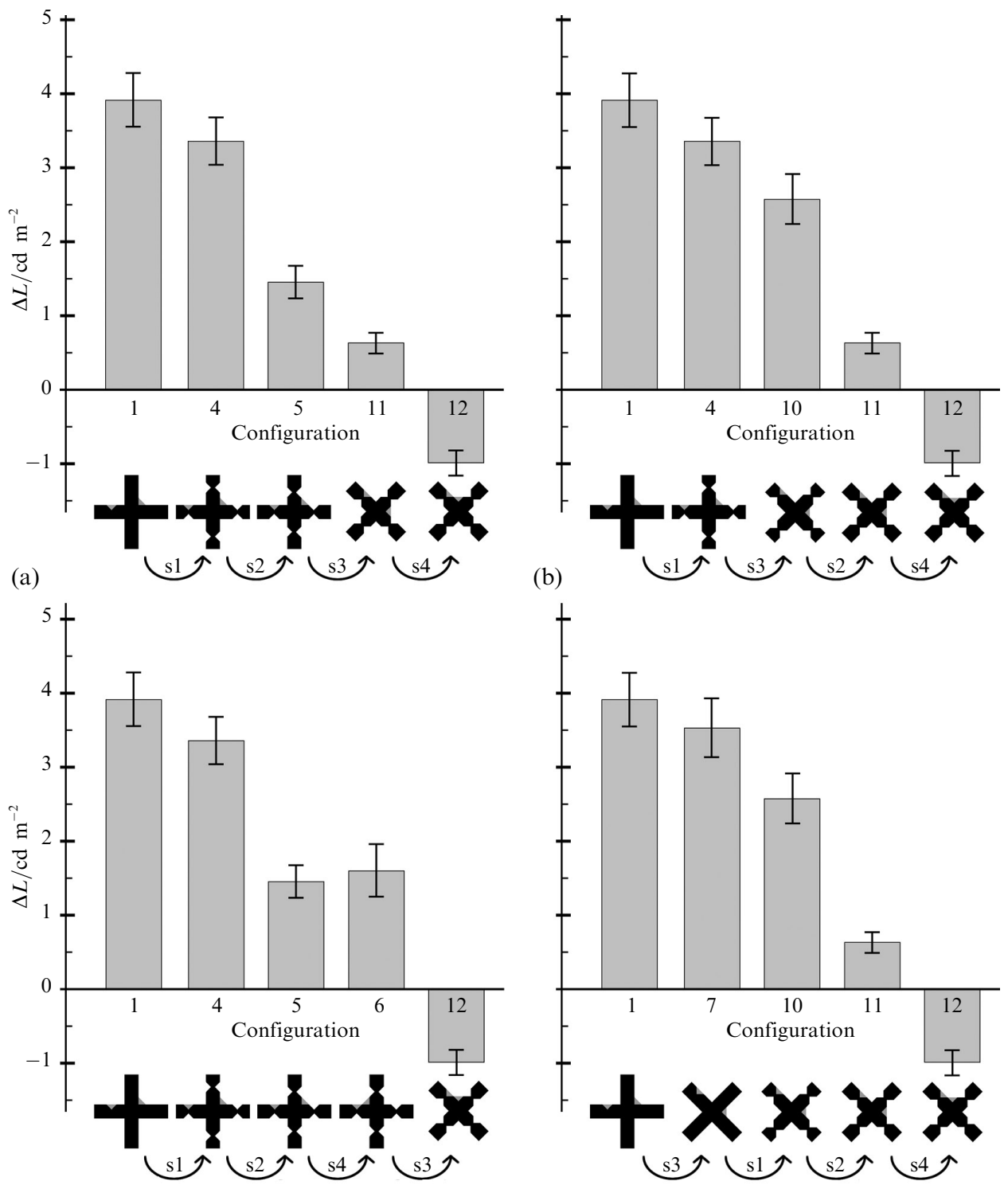

(c)

(d)

Figure 6. Four different routes according to which the original Benary cross illusion can be altered in a stepwise fashion. Arrows $\mathrm{s} 1-\mathrm{s} 4$ represent the different types of manipulations. s1: making indentations to the cross; s2: aligning contours of the right grey triangle and the indentations; s3: rotating the configuration by $45^{\circ}$; s4: repositioning the right grey triangle so that the contours of both triangles are aligned. With the exception of manipulation s4 in (c), each panel shows a decrease of the effect after each further manipulation of the configuration. The order in which the configuration is manipulated with respect to the experimental factors is different in each panel, which explains why slightly different patterns can be observed for each panel. Error bars represent \pm 1 SEM.

cross on the perceived brightness. A paired-samples $t$-test between configurations 1 and 4 (figures $6 a-6 c$ ) reveals that the effect induced by the original cross is significantly larger than the effect resulting from making indentations to the cross $\left(t_{15}=2.18, p<0.05\right)$. The decrease in brightness differences as a result of making additional indentations to the cross was also found when comparing configurations 7 and 10 (figure $6 \mathrm{~d} ; t_{15}=3.96, p<0.005$ ). Next, we compare the effect after making 
indentations to the cross with the residual effect after aligning these indentations with the hypotenuse of the right triangle (s2). This comparison gives us information on the relative contribution of T-junctions on the perceived brightness of the right grey triangle in the Benary cross. Note that the new junctions may favour an interpretation where the triangle is no longer juxtaposed to the cross-like figure, but superimposed on that figure (with the black part of the cross-like figure continuing underneath the triangle; see also section 4 for a further elaboration on this possibility). The decrease in brightness difference from configuration 4 to configuration 5 is relatively large (figures $6 \mathrm{a}$ and $\left.6 \mathrm{~b}: t_{15}=6.49, p<0.001\right)$. A similar result was obtained when comparing the effect of configurations 10 and 11 (figures $6 \mathrm{~b}$ and $6 \mathrm{~d}$ : $t_{15}=6.68, p<0.001$ ). Subsequently, we looked at the effect of rotating the configuration by $45^{\circ}$ (s3) by comparing rightside-up configurations with the rotated versions in each of the panels of figure 6 . The comparison between configurations 5 and 11 (figure 6a) revealed a significant difference $\left(t_{15}=4.11, p<0.005\right)$; similar results were obtained for configurations 4 and 10 (figure 6b): $t_{15}=3.67, p<0.005$, and for configurations 6 and 12 (figure $6 \mathrm{c}$ ): $t_{15}=5.50$, $p<0.001$; the comparison between configurations 1 and 7 (figure 6d) was not significant: $t_{15}=1.63, p=0.123$. Finally, we consider the change in position of the right triangle (s4). This repositioning resulted in a reduction of the original effect. The original effect was even reversed, in which the right triangle now looked lighter than the left triangle. The pairwise comparisons of the configurations were all significant (for configurations 11 and 12; figures $6 \mathrm{a}, 6 \mathrm{~b}, 6 \mathrm{~d}: t_{15}=7.15, p<0.001$ ) (for configurations 6 and 12; figure $6 \mathrm{c}$ : $\left.t_{15}=5.50, p<0.001\right)$.

\subsection{Control observations}

To be confident that the effect of orientation we found is not the result of a bias caused by the CRT monitor, we performed a straightforward control measurement with stimuli printed on paper. We presented configurations 6 and 12 (see figure 4) to ten naive subjects. We chose configurations 6 and 12, as the orientation difference between these configurations appeared to be highly critical with respect to the direction of the brightness difference; in configuration 6 (the upright cross) the left triangle is perceived as brighter, whereas in configuration 12 (the oblique cross) the right triangle is seen as brighter (see also figure 6c). In fact, we had one single printed configuration which was presented to each subject once in its upright orientation (configuration 6, figure 4). and ones rotated by $45^{\circ}$ (configuration 12, figure 4). Participants did not know that they were shown the same paper twice. Furthermore, the order in which the stimuli were presented was counterbalanced across participants. For both stimuli we asked the subjects to judge which of the two triangles appeared lighter-either the left or the right. In its upright position all ten subjects reported that the left triangle looked brighter than the right triangle, but when the paper was rotated by $45^{\circ}$, nine out of the ten subjects reported that the right triangle looked brighter than the left triangle. These results are in line with the results obtained on the CRT and therefore give us confidence that the effect of orientation found in the original experiment was not simply due to an orientation-dependent monitor bias.

\section{General discussion}

The aim of this study was to disentangle the influence of several factors in causing brightness differences in the Benary cross. By varying differences in contour junctions with areas surrounding the triangles as well as more global factors, like the symmetry of the figure and its orientation, we were able to study their influence on perceived brightness differences. Multiscale spatial-filtering approaches (Blakeslee and McCourt 2001; Robinson et al 2007) have successfully predicted the direction of the Benary effect. Although some of the effects we have presented might be accounted for by response 
normalisation, the effect of orientation we present in this study seems to pose a serious challenge to these models. Both Blakeslee and McCourt's ODOG model and Robinson et al's extension of the model (FLODOG) do not differentiate in perceived brightness prediction for different orientations of a visual display. This challenge will have to be overcome to give these and similar models even more theoretical impact.

Although the results we present are rather clear-cut, it seems hard to indicate what the impact of our current data is for conceptual models like Gilchrist et al's anchoring theory (1999) and Anderson's scission theory (1997). Using Gilchrist et al's terminology, one could argue that our manipulations of the cross change the frame of reference, either locally or globally, which determines which surfaces belong to each other. Since the rules defining the frame of reference are not very-well defined within their theory, it seems difficult to use our stimuli to test their theory. Scission theory relies mainly on the role that local junctions play in determining different layers. The principle of scission might well play a role in determining a surface's perceived brightness, also in the Benary cross. However, by changing the configuration as we did in our stimuli, other factors like the preference for symmetrical shapes might interact with the effects of local scission, which according to Anderson is largely determined by the junctions surrounding a surface.

To get a perspective on the role of the investigated factors in terms of figurebackground organisation, we first return to some effects of the individual manipulations, following the route as described in figure 6a. After that, we give a more detailed analysis of the effect of our manipulations on the current brightness phenomena. We will focus here on the positional ambiguity of the left and right triangle in relation with the dark-grey cross and speculate on the effect of the manipulations on that ambiguity. In the original Benary cross the left triangle is most likely seen to be superimposed on the cross, whereas the right triangle is most likely seen to be juxtaposed to the cross. For all manipulations, the position of the triangles, which is either perceived as juxtaposed to or superimposed on the cross-like shape, seems to play a decisive role in the relative-brightness judgments.

First, when we compare the effect in the original Benary cross (configuration 1) with the effect after making indentations to the cross (configuration 4), we see a significant reduction of brightness differences between the triangles. Following Gestalt assumptions on preferences for regular shapes (eg Wertheimer based on symmetry, van Lier et al 1995), an interpretation is plausible in which the left triangle is no longer perceived as superimposed on the dark cross-like shape but as lying on the white background, juxtaposed to a symmetrically indented cross. The alternative figure-ground interpretation makes the triangle appear darker than in the original configuration. This result supports the hypothesis that the global interpretation of the figure plays an important role in determining locally perceived brightness, which is in line with literature suggesting that perceptual organisation plays a role in determining perceived brightness (eg Agostini and Galmonte 2002; Agostini and Proffitt 1993).

Next, we look at the effect of shifting the indentations in such a way that the right triangle is aligned with the contours of the cross (configuration 5). As a result of this manipulation, local contour junctions between triangles and cross-like figure change. The alignment of the contours results in increased ambiguity regarding the positioning of the right triangle, as now the right triangle could more easily be perceived to be superimposed on the dark cross-like shape. This increased ambiguity regarding the background of the right triangle would lead to a perception of that triangle as being lighter than in the previous configuration. The results show that this manipulation indeed led to a dramatic reduction in brightness differences between the triangles, which provides additional support for the idea that T-junctions play an important role in determining perceived brightness (eg Anderson 1997; Todorović 1997; Zaidi et al 1997). 
Rotating the figure by $45^{\circ}$ (configuration 11), again led to a significant reduction of brightness differences between the two triangles. Note that our visual system has a preference for horizontal and vertical orientations of contours (eg Campbell et al 1966; Furmanski and Engel 2000; Orban et al 1984). For these reasons we speculate that the contour orientations increased the plausibility that the right triangle was perceived to be superimposed on the cross-like shape. The role of the positional ambiguity of the right triangle is further supported by a significant 2-way interaction between the factors indentations and orientation. This interaction shows that brightness differences after rotation are reduced more for configurations with indentations than for configurations without indentations.

The final manipulation in the route described in figure 6a concerns relocating the right triangle to the upper corner of the intersection of the cross (configuration 12). As a result of this relocation, the original effect is reversed. The reversal from configuration 11 to 12 could be explained by the human preference for vertical symmetries (eg Palmer and Hemenway 1978; Sekuler 1994; Wagemans 1995; Wenderoth 1994). Based on this preference, an interpretation in configuration 11 in which the right grey triangle is perceived as being juxtaposed to the cross is most likely. After relocating this triangle to the upper corner of the intersection of the cross, the interpretation becomes more ambiguous, since the cross is now vertically symmetrical for both a juxtaposition and a superposition interpretation of the right grey triangle. Note that a similar decrease in brightness differences does not occur from configuration 5 to configuration 6 (see figure $6 \mathrm{c}$ ), when repositioning of the right triangle takes place before rotation of the configuration, which only strengthens our speculations about the influence of orientations of symmetries and contours on the perceived interpretations.

To get a further grip on the influence of the various manipulations on the perceived brightness differences we have performed an additional analysis. In this analysis we make use of the assumption that figure background organisation is an important mediating factor in the Benary effect: a triangle perceived on the black cross-like figure tends to look brighter than a triangle perceived on the white background. We now consider four possible interpretations for each configuration: (i) the left triangle is superimposed on the black cross-like shape, the right triangle is juxtaposed to that shape; (ii) the left and right triangles are both juxtaposed to the cross-like shape; (iii) the left triangle is juxtaposed to the cross-like shape; the right triangle is superimposed on that shape; (iv) the left and right triangles are both superimposed on the cross-like shape. These four conditions would lead to the following brightness judgments, respectively: (i) the left triangle is brighter; (ii) triangles have equal brightness; (iii) the right triangle is brighter; (iv) the triangles have equal brightness.

Next, we consider the possible interpretations for the outlined figure-ground segregations. In figure 7, we have drawn the corresponding shapes for each figureground segregation (notice that for configurations 1 and 2, and configurations 7 and 8 , we consider just one exemplar, as these configurations do not structurally differ from each other). For example, in figure 7 top left (upper row), the original Benary cross is shown; in the same row, the four possible interpretations in accordance with the four above assumptions are shown. For each of these interpretations we evaluate whether it is supported by three figural properties.

(i) Global symmetry. Interpretations comprising regular shapes may be more prevalent akin to the Gestalt notion of Prägnanz (ie a general preference for interpretations that comprise 'good shapes' - Wertheimer 1923), later reformulated into the so-called minimum principle (eg Hochberg and McAlister 1953; Leeuwenberg 1969). Various kinds of regularities may increase a shape's preference, but we will focus here on the occurrence of global symmetry where shapes have at least one axis of symmetry (for a review on 


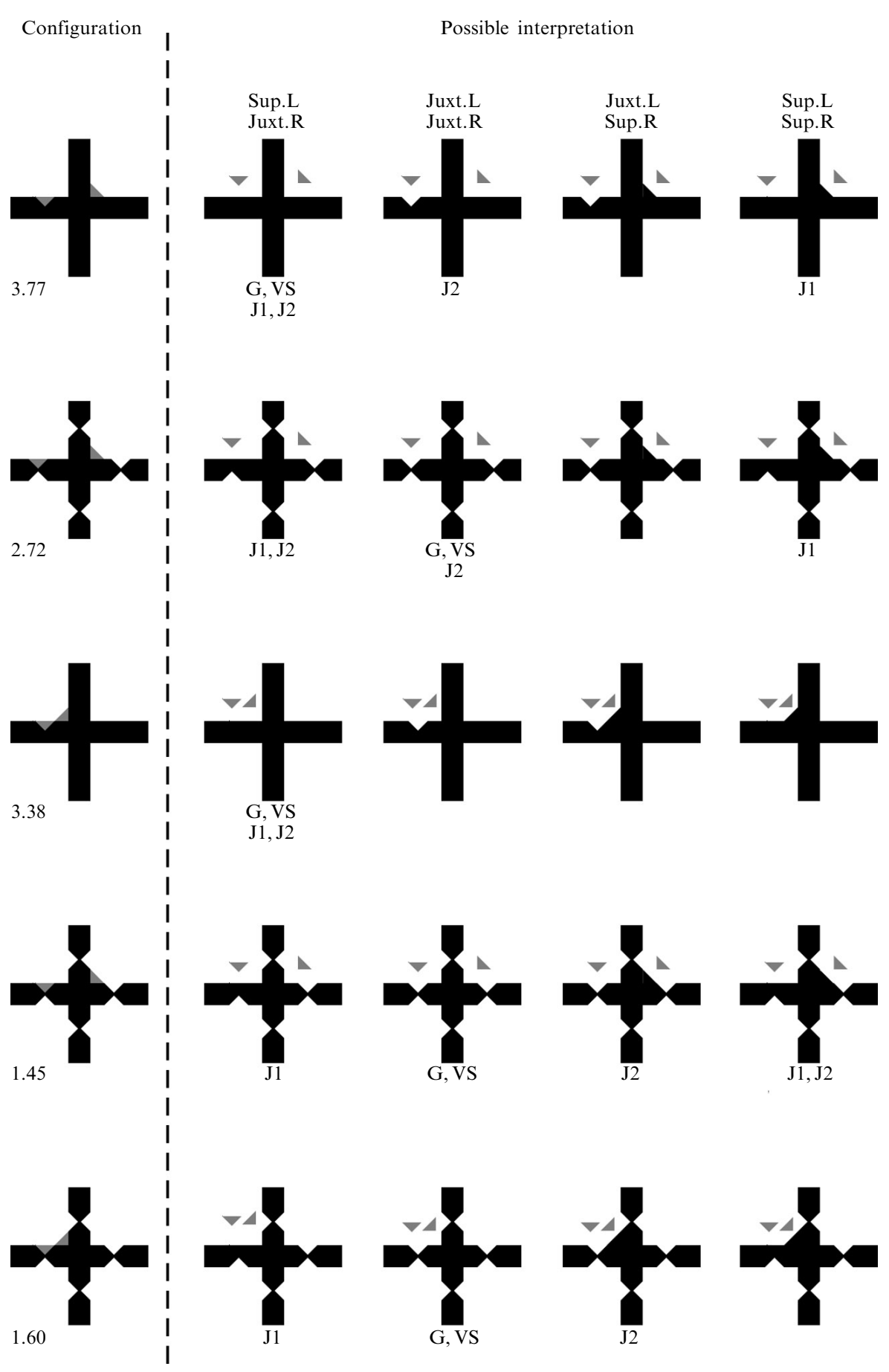

Figure 7. For each of our test configurations (left columns), 4 different interpretations are displayed, according to four possible figure-ground segregations. Sup.L: superposition left triangle; Juxt.L: juxtaposition left triangle; Sup.R: superposition right triangle; Juxt.R: juxtaposition right triangle. Various figural properties that support the specific interpretations are indicated below each interpretation G: global symmetry, VS: vertical orientation axis of symmetry, J1: local junctions of left triangle support collinearity of the contour of the cross, J2: local junctions of right triangle support collinearity of the contour of the cross. The numbers in the configuration columns refer to the $\Delta L$ values as measured in the experiment. 


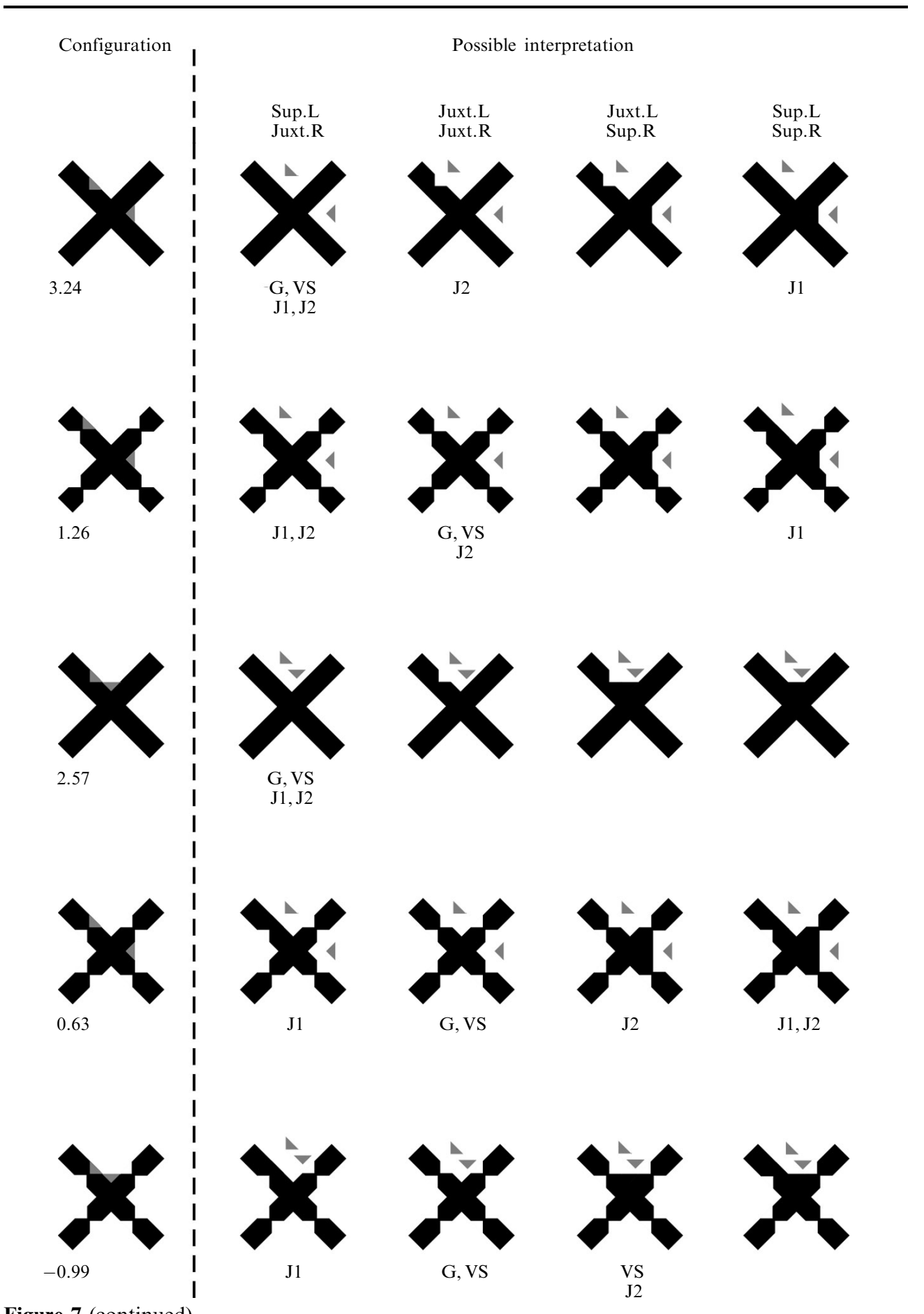

Figure 7 (continued).

the impact of bilateral symmetry see eg Wagemans 1995). In figure 7, all interpretations comprising such bilateral symmetrical cross-like shapes are labelled with letter ' $G$ '.

(ii) Vertical orientation of axis of symmetry. Whereas the previous property deals with orientation-independent aspects of an interpretation, this property deals with an orientation-dependent aspect. Earlier research has shown preferences especially for 
vertical symmetries (see eg Sekuler 1994; Wagemans 1995; Wenderoth 1994). In figure 7, all interpretations for which an axis of symmetry is vertical have been labelled 'VS'.

(iii) Junction type. Next we consider the connection between the triangles and the crosslike shapes. We reason that the stronger the connection between contours of different shapes within a certain interpretation, the less likely that interpretation will occur. This idea is akin to the 'avoidance-of-coincidence' principle (Rock 1983). It is argued elsewhere (eg van der Helm 2000; van Lier et al 1994) that the strength of the connection between the contours in a local junction can be determined by comparing the minimal number of parameters necessary to describe the actual junction with the minimal number of parameters necessary to describe the disconnected contours. In the present study, the crucial junctions are those where three luminance fields come together. For each of these crucial junctions two interpretations are possible: (a) the contour of the cross is 'hard-edged', making a $45^{\circ}$ turn at the junction, or (b) the orientation of the contour of the cross is constant (collinear) across the junction. In line with the above-mentioned studies, we consider the latter junctions to be less coincidental (van Lier et al 1994). Therefore, interpretations presuming the latter type of junctions are more prevalent than interpretations presuming the first type of junctions. In figure 7, an interpretation has been labelled ' $\mathrm{J} 1$ ' when both crucial junctions (ie connecting three luminance fields) of the left triangle reveal orientation constancy of the contour of the cross and, similarly, an interpretation has been labelled ' $J 2$ ' when both crucial junctions of the right triangle reveal orientation constancy of the contour of the cross.

To illustrate how the above figural properties support one or the other interpretation, we consider the first configuration in figure 7 (left panel, top; ie the original Benary cross) and the last configuration in figure 7 (right panel, bottom). For the original Benary cross, the first interpretation (in which the left triangle is superposed on the cross and the right triangle is juxtaposed to the cross) is supported by most figural properties. More specifically, this interpretation (figure 7, column 1) is supported by 4 properties (labelled $\mathrm{G}, \mathrm{VS}, \mathrm{J} 1$, and $\mathrm{J}$ ), while the other suggested interpretations of this configuration (figure 7, columns 2 to 4 ) are supported by 1 or 0 properties (note that column numbers $1-4$ refer to the four columns representing the 'possible interpretations' in the left and right panels). The configuration at the bottom right of figure 7 shows a completely different distribution of supporting properties. Here, the interpretation of column 1 (in which the left triangle is superposed on the cross and the right triangle is juxtaposed to the cross) is supported by just 1 of the discussed figural properties (J1), whereas the interpretations in column 2 (juxtaposition left triangle, juxtaposition right triangle), and column 3 (juxtaposition left triangle, superposition right triangle) are both supported by 2 figural properties (and the interpretation in column 4 by 0 properties).

The differential support for the various interpretations to a certain extent seems to fit the notion that a superposition triggers the perception of a brighter triangle, whereas a juxtaposition would trigger the perception of less bright triangle. To further explore this idea, we have correlated the measured brightness difference between the left and right triangle in the configuration $(\Delta L$, table 1$)$ with the total number of the supporting figural properties $(\mathrm{G}, \mathrm{VS}, \mathrm{J} 1, \mathrm{~J} 2)$ for each of the four interpretations as shown in figure 7 (note that we have taken the mean $\Delta L$ for the similar configurations $1-2$ and $7-8$ of figure 4). Pearson correlations between the measured effect size $(\Delta L)$ and the number of supporting properties as indicated in the four columns reveals the following results. Column 1 (left superposition, right juxtaposition): $R=0.817$, $p<0.005$; column 2 (left juxtaposition, right juxtaposition): $R=-0.500$, ns; column 3 (left juxtaposition, right superposition): $R=-0.872, p<0.005$; column 4 (left superposition, right superposition): $R=-0.053$, ns. Finally, correlating the $\Delta L$ values with the difference in the number of supporting properties between column 1 (left superposition, right juxtaposition) and column 3 (left juxtaposition, right superposition) reveals: 
$R=0.882, p<0.005$. The above correlations support the notion that brightness differences depend on the figural properties mentioned here. The higher the number of figural properties that support the interpretation of superposition of the left triangle and juxtaposition of the right triangle, the greater the effect size (left triangle brighter than right triangle) for a certain configuration. On the other hand, the higher the amount of figural properties supporting the interpretation of juxtaposition of the left triangle and superposition of the right triangle, the smaller the effect size for a certain configuration.

The results of this additional analysis support the idea that perceptual organisation is a mediating factor with respect to the perceived brightness differences in the Benary cross. Note, however, that this cannot be regarded as a final explanatory account. As mentioned above, we have focused on a rather small subset of figural properties that support one or the other interpretation and we have simply correlated $\Delta L$ with the number of such properties supporting one or the other interpretation. Subsequent analyses may further apply weighing factors to the various properties or may take account of other properties as well. For example, the analysis does not appropriately account for the effect of orientation found in the present study. One could speculate that the orientation effect on the brightness difference might be (partly) due to the visual system's reference for horizontal and vertical contours as compared to oblique contours (eg DeValois and DeValois 1988; Mansfield and Ronner 1978). In that case, the differential processing of collinear contours in the upright and oblique oriented configurations would legitimate a larger weight for the factors ' $\mathrm{J} 1$ ' and ' $\mathrm{J} 2$ ' when the contours of the cross are horizontal or vertical. For example, if we would account for the occurrence of such horizontal or vertical contours as additional properties (let us say, $\mathrm{J}^{*}$ and $\mathrm{J} 2 *$ ), then the results of the above tentative analysis would further improve. In figure 7, the distribution of supporting properties would be more in line with brightness measurements; note that in the left panel of figure 7 most $\mathrm{J} 1$ and $\mathrm{J} 2$ classifications largely comply with either horizontal or vertical contours of the cross and could be marked as $\mathrm{J}^{*}$ and $\mathrm{J}_{2}^{*}$, whereas in the right panel this is the other way round. An analysis, similar to the one above, correlating $\Delta L$ with the difference between (i) the number of properties supporting a left superposition, right juxtaposition interpretation (column 1) and (ii) the number of properties supporting a left juxtaposition, right superposition interpretation (column 3), would reveal a Pearson correlation of $R=0.961(p<0.001)$.

Altogether, the results demonstrate that the perceived brightness differences between the triangular patches in the Benary cross can be modulated by making various figural changes. These changes are suggested to influence preferred figure-ground organisation and, with that, perceived brightness. The figure-ground organisation is quite stable in the original Benary cross (superposition of the left triangle, juxtaposition of the right triangle) but may significantly change as a result of various stimulus manipulations. The current results support the idea that global and local properties (such as symmetry and junction types) but also orientation independence and orientation dependence of these properties determine the brightness differences in the Benary cross.

Acknowledgments. MV was supported by NWO grant 400-03-406, awarded to RVL. We thank two anonymous reviewers for their valuable comments on a previous version of the manuscript.

\section{References}

Adelson E H, 1993 "Perceptual organization and the judgement of brightness" Science 2622042 - 2044

Agostini T, Galmonte A, 2002 "Perceptual organization overcomes the effects of local surround in determining simultaneous lightness contrast" Psychological Science 13 89-93

Agostini T, Proffitt D R, 1993 "Perceptual organization evokes simultaneous lightness contrast" Perception $22263-272$

Anderson B L, 1997 "A theory of illusory lightness and transparency in monocular and binocular images: the role of contour junctions" Perception $26419-453$ 
Anderson B L, 2003 "The role of occlusion in the perception of depth, lightness, and opacity" Psychological Review $110785-801$

Benary W, 1924 "Beobachtungen zu einem Experiment über Heligkeitskontrast" Psychologische Forschung $5131-142$

Blakeslee B, McCourt M E, 1999 "A multiscale spatial filtering account of the White effect, simultaneous brightness contrast and grating induction" Vision Research 394361 - 4377

Blakeslee B, McCourt M E, 2001 "A multiscale spatial filtering account of the Wertheimer-Benary effect and the corrugated Mondrian" Vision Research 41 2487-2502

Campbell F W, Kulikowski J J, Levinson J, 1966 "Effect of orientation on visual resolution of gratings" Journal of Physiology $18427-436$

Coren S, 1969 "Brightness contrast as a function of figure-ground relations" Journal of Experimental Psychology $80517-524$

DeValois R L, DeValois K K, 1988 Spatial Vision (New York: Oxford University Press)

Furmanski C S, Engel S A, 2000 "An oblique effect in human primary visual cortex" Nature Neuroscience $3535-536$

Gilchrist A, Kossyfidis C, Bonato F, Agostini T, Cataliotti J, Li X, 1999 "An anchoring theory of lightness perception" Psychological Review $106795-834$

Helm P A van der, 2000 "Simplicity versus likelihood in visual perception: from surprisals to precisals" Psychological Bulletin $126770-800$

Hochberg J, McAlister E, 1953 "A quantitative approach to figural 'goodness" "Journal of Experimental Psychology 46361 - 364

Howe P D L, 2005 "White's effect: Removing the junctions but preserving the strength of the illusion" Perception $34557-564$

Leeuwenberg E, 1969 "Quantitative specification of information in sequential patterns" Psychological Review $76216-220$

Lier R van, Helm P van der, Leeuwenberg E, 1994 "Integrating global and local aspects of visual occlusion" Perception $23883-903$

Lier R J van, Helm P A van der, Leeuwenberg E L J, 1995 "Competing global and local completions in visual occlusion" Journal of Experimental Psychology: Human Perception and Performance $21571-583$

Mansfield R J, Ronner S F, 1978 "Orientation anisotropy in monkey visual cortex" Brain Research $149229-234$

Oh S, Kim J-O, 2004 "The effects of global grouping laws on surface lightness perception" Perception \& Psychophysics $66792-799$

Orban G A, Vandenbussche E, Vogels R, 1984 "Human orientation discrimination tested with long stimuli" Vision Research $24121-128$

Palmer S E, Hemenway K, 1978 "Orientation and symmetry-effects of multiple, rotational, and near symmetries" Journal of Experimental Psychology: Human Perception and Performance 4691 - 702

Robinson A E, Hammon P S, Sa R de, 2007 "Explaining brightness illusions using spatial filtering and local response normalization" Vision Research $471631-1644$

Rock I, 1983 The Logic of Perception (Cambridge, MA: MIT Press)

Sekuler A B, 1994 "Local and global minima in visual completion: Effects of symmetry and orientation" Perception $23529-545$

Todorović D, 1997 "Lightness and junctions" Perception 26 379-394

Vergeer M, Lier R van, 2010 "Capturing lightness between contours" Perception 391565 - 1578

Wagemans J, 1995 "Detection of visual symmetries" Spatial Vision 9 9-32

Wallach H, 1948 "Brightness constancy and the nature of achromatic colors" Journal of Experimental Psychology 38 310-324

Wenderoth P, 1994 "The salience of vertical symmetry" Perception 23221 -236

Wertheimer M, 1923 "Untersuchungen zur Lehre von der Gestalt" Psychologische Forschung 4301 - 350

White M, 1979 "A new effect of pattern on perceived lightness" Perception 8 413-416

Zaidi Q, Spehar B, Shy M, 1997 "Induced effects of backgrounds and foregrounds in threedimensional configurations: the role of T-junctions" Perception 26 395-408 


\section{PERCEPTION}

VOLUME 402011

www.perceptionweb.com

Conditions of use. This article may be downloaded from the Perception website for personal research by members of subscribing organisations. Authors are entitled to distribute their own article (in printed form or by e-mail) to up to 50 people. This PDF may not be placed on any website (or other online distribution system) without permission of the publisher. 